\title{
Measurement Models for Time Series Analysis: Estimating Dynamic Linear Errors-in-Variables Models
}

\author{
Gregory E. McAvoy
}

\begin{abstract}
This article uses state space modeling and Kalman filtering to estimate a dynamic linear errors-in-variables model with random measurement error in both the dependent and independent variables. I begin with a general description of the dynamic errors-in-variables model, translate it into state space form, and show how it can be estimated via the Kalman filter. I report the results of a simulation in which the amount of random measurement error is varied, to demonstrate the importance of estimating measurement error models and the superiority that Kalman filtering has over regression. I use the model in a substantive example to examine the effects of public opinion regarding nuclear power on the enforcement decisions of the Nuclear Regulatory Commission. I then estimate a dynamic linear errors-in-variables model using multiple indicators for the latent variables and compare simulations of this model to the single indicator model. Finally, I provide substantive examples which examine the effect of people's economic expectations on their approval of the president and their approval of government more generally.
\end{abstract}

Earlier versions of this article were presented at the 1995 Annual Meeting of the American Political Science Association, Chicago, Illinois, and the 1996 Annual Political Methodology Summer Conference, Ann Arbor, Michigan. I would like to thank the following people for helpful comments and/or conversations: Susan Bickford, Suzanna DeBoef, Donald Green, Jim Stimson, George Rabinowitz, and Renée Smith. 


\section{Introduction}

The topic of measurement errors in time series analysis periodically captures the attention of economists and statisticians, but such attention is rarely sustained. This episodic history is explained by analysts' concern for other problems in time series analysis (like the detection and correction of problems in the disturbance term) and the difficulty of identifying and estimating those measurement models that have been proposed (e.g., Geweke 1977; Maravall and Aigner 1977; Aigner, Hsiao, Kapteyn and Wansbeek 1984). Political scientists, however, do not have the luxury of ignoring measurement error since many of our important and interesting concepts are not directly observable or their measures are contaminated with random error. The tendency to ignore measurement issues in time series analysis appears to be diminishing as the methods to conduct this type of analysis - state space modeling and Kalman filtering-become more prevalent and computer software and hardware make estimation easier. ${ }^{1}$

Within political science, those addressing measurement issues in time series have focused on the estimation of models with error in the dependent variable. Beck (1990) describes state space modeling and Kalman filtering and demonstrates the utility of these tools for the estimation of random measurement error and dynamic factor analytic models. Kellstedt, McAvoy and Stimson (1996) show the efficiency gains from estimating a measurement model for the dependent variable and the precision of Kalman filter estimates in the face of autocorrelation. These measurement techniques have been applied to a variety of substantive problems, including dynamic representation, racial inequality, monetary policy, public entrepreneurship, and arms races. ${ }^{2}$

Measurement error is not limited to the dependent variables, however. The consequences of measurement error in the independent variables are of equal, if not greater, importance. As we know from any textbook treatment of measurement error, random error in the independent variables produces biased and inconsistent ordinary least squares (OLS) estimates.

This paper uses state space modeling and Kalman filtering to es-

\footnotetext{
${ }^{1}$ The simulations in this article were run on a UNIX platform using Rats 4.1. The substantive applications were run on a PC using GAUSS 3.2 and the maximum likelihood module, version 4.0. The programs to produce the tables in this article are available via the Internet at $\mathrm{http}: / /$ www.uncg.edu/ gemcavoy.

${ }^{2}$ Williams and McGinnis (1992) conduct a dynamic factor analysis to estimate superpower rivalry. They use spectral techniques to estimate their models, rather than Kalman filtering as used by Beck (1990) and Kellstedt, McAvoy and Stimson (1996).
} 
timate a dynamic linear errors-in-variables model with random measurement error in both the dependent and independent variables. I begin with a general description of the dynamic errors-in-variables model, translate it into state space form, and show how it can be estimated using the Kalman filter. Then I report the results of a simulation in which the amount of random measurement error is varied and, thus, demonstrate the importance of estimating measurement error models and the superiority that Kalman filtering has over regression. Next I use the model in a substantive example to examine the effects of public opinion regarding nuclear power on the enforcement decisions of the $\mathrm{Nu}$ clear Regulatory Commission. I then estimate a dynamic linear errorsin-variables model using multiple indicators for the latent variables and compare simulations of this model to the single indicator model. Finally, I provide substantive examples that examine the effect of people's economic expectations on their approval of the president and their approval of government more generally.

\section{The Dynamic Linear Errors-in-Variables Model}

The dynamic linear errors-in-variables model with a single indicator for each latent variable is a useful starting point from which to investigate dynamic latent variables. The single indicator model has been described as a dynamic shockerror model. The general model as described in Aigner, Hsiao, Kapteyn and Wansbeek (1984) and Ghosh (1989) is

$$
\begin{aligned}
x_{t}= & \beta_{1} x_{t-1}+\beta_{2} x_{t-2}+\cdots+\beta_{p} x_{t-p} \\
& +\gamma_{0} u_{t}+\gamma_{1} u_{t-2}+\cdots+\gamma_{q} u_{t-q}+\epsilon_{t},
\end{aligned}
$$

where $p$ is the lag of the endogenous variable, and $q$ is the lag of the exogenous variable. This is a single-equation model with lagged endogenous and exogenous variables, which are assumed to be stationary. The equation disturbance term, $\epsilon_{t}$, constitutes the "shock" in this model. The variables $x_{t}$ and $u_{t}$ are not directly observable due to random measurement errors. The measurement equations for these variables are

$$
\begin{aligned}
& x_{t}^{*}=x_{t}+v_{x t} \\
& u_{t}^{*}=u_{t}+v_{u t},
\end{aligned}
$$

where $x_{i}^{*}$ and $u_{i}^{*}$ are the observed variables, and $v_{x t}$ and $v_{u t}$ are random measurement errors. The error terms, $v_{x t}$ and $v_{u t}$, and the shock, $\epsilon_{t}$, are assumed to be normally distributed, mutually independent, white noise processes. OLS estimation of equation (1) using the observed variables leads to biased and inconsistent estimates due to the measurement error 
in the independent variable. The bias is the result of contemporaneous correlation between the regressor and the error term, the classic errorsin-variables problem.

In order to overcome the errors-in-variables problem, these equations must be estimated simultaneously. Therefore, one must be sure that the specified model is identified. Maravall (1979) provides proofs for the identification of a number of variants of the dynamic shock-error model, including the ones used here. Recently some economists have developed counting rules for identification, ones that are similar to the rank and order conditions used to check for identification in simultaneous equation models (e.g., Solo 1986; Nowak 1993). ${ }^{3}$

I will work with a simplified version of the model described above,

$$
x_{t}=\beta_{1} x_{t-1}+\gamma_{0} u_{t}+\epsilon_{t}
$$

but one that imposes some structure on the exogenous variable

$$
u_{t}=\lambda_{1} u_{t-1}+e_{t} \text {. }
$$

This additional equation helps insure that the model is identified and makes sense substantively for many issues. It is not necessary to include this additional equation for identification of the system, but without it, we must have $p>2$ and $q>1$ or $p>1$ and $q>2$ (Ghosh 1989).

Estimation of this model requires writing the four equations in state space form, specifying the appropriate Kalman filtering equations, and maximizing the likelihood function. The dynamic errors-in-variables model proposed here differs slightly from other political science applications (Beck 1990; Kellstedt, McAvoy and Stimson 1996) because there are two state vectors, each with its own measurement equation. To put equations (2) and (3) into state space form requires some algebraic manipulation to insure that only lagged values of the state vector appear on the right hand side of the transition equations. Substituting equation (3) into equation (2) and then representing the two equations in matrix notation yields the "state" or transition equations

$$
\left[\begin{array}{l}
u_{t} \\
x_{t}
\end{array}\right]=\left[\begin{array}{ll}
\lambda_{1} & 0 \\
\gamma_{0} \lambda_{1} & \beta_{1}
\end{array}\right]\left[\begin{array}{l}
u_{t-1} \\
x_{t-1}
\end{array}\right]+\left[\begin{array}{ll}
1 & 0 \\
\gamma_{0} & 1
\end{array}\right]\left[\begin{array}{l}
e_{t} \\
\epsilon_{t}
\end{array}\right] .
$$

The measurement equations can also be written in matrix notation,

$$
\left[\begin{array}{l}
u_{t}^{*} \\
x_{t}^{*}
\end{array}\right]=\left[\begin{array}{l}
u_{t} \\
x_{t}
\end{array}\right]+\left[\begin{array}{l}
v_{u t} \\
v_{x t}
\end{array}\right]
$$

\footnotetext{
${ }^{3}$ For additional information on the identification of dynamic linear errors-invariables, see Nowak (1993) and Scherrer, Deistler, Kopel and Reitgruber (1991). For more information on the estimation of these models, see Aoki (1990) and Lomba (1990).
} 
With the measurement and transition vectors in state space form, the dynamic linear errors-in-variables model can easily be incorporated into the Kalman filter for estimation. The Kalman filter is a recursive process that uses information from the previous time period to form a conditional forecast of the state vectors and calculates the prediction error from the measurement equations. The complete set of Kalman equations for this analysis is described in the Appendix. The critical calculations derived recursively from the filter are the estimates of the state vector $\left(\mathbf{X}_{t}\right)$, a vector of prediction errors or innovations at $t\left(\eta_{t}\right)$, and the covariance matrix of innovations conditional on information at $t-1\left(\mathbf{H}_{t}\right)$. With this information, the log-likelihood function for the dynamic linear errors-in-variables model can be calculated:

$$
L\left(\lambda, \beta, \gamma, e, \epsilon, v_{u}, v_{x}\right)=-\frac{1}{2} \sum_{t=1}^{T} \log \operatorname{det} \mathbf{H}_{t}-\frac{1}{2} \sum_{t=1}^{T} \eta_{t}^{\prime} \mathbf{H}_{t}^{-1} \eta_{t} .
$$

Because it depends on conditional innovations at time $t$, this specification is often described as the prediction error decomposition form of the likelihood (Harvey 1989; Ghosh 1989). The likelihood function can be maximized with respect to the transition and measurement parameters $\left(\lambda, \beta, \gamma, e, \epsilon, v_{u}, v_{x}\right)$ to get the maximum likelihood estimates."

\section{Random Measurement Error Simulation}

Simulated data are useful for this comparison of OLS and dynamic linear errors-in-variables because the true parameter estimates are known, but more importantly, because the amount of random error can be manipulated. I constructed data sets based on three different scenarios, each with a different amount of error in the observed variables. Each scenario was simulated 100 times and the model estimated by OLS and the Kalman filter. The average of the parameter estimates for each scenario provides the basis for a meaningful comparison between OLS and the maximum likelihood estimates of the Kalman filter.

The true parameters for the simulation are

$$
\lambda_{1}=.88, \quad \beta_{1}=.65, \quad \gamma_{0}=.27, \quad \gamma_{1}=.52 \text {. }
$$

Here, $\gamma_{1}$ is the coefficient of an exogenous variable measured without error. The error variances for the transition equations were set at 5.0.

\footnotetext{
${ }^{4}$ Ghosh (1989) and Watson and Engle (1983) use the EM algorithm to maximize the function because the EM equations are calculated to insure positive estimates of the error variances. However, it is a much more cumbersome to estimate the likelihood function using the EM method. Here, I use the generalized maximum likelihood estimator procedures in GAUSS and RATS and did not encounter any problems with negative variances.
} 
TABLE 1. Summery Estimates for Simulations with Measurement Error Varied

\begin{tabular}{lccc}
\hline Error & Case 1 & Case 2 & Case 3 \\
$\sigma_{v 1}=\sigma_{v 2}=$ & 1.0 & 5.0 & 15.00
\end{tabular}

Maximum Likelihood Estimates

$\begin{array}{lcccccc} & \text { Mean } & \text { SD } & \text { Mean } & \text { SD } & \text { Mean } & \text { SD } \\ \hat{\lambda}_{1} & 0.885 & 0.016 & 0.870 & 0.025 & 0.876 & 0.034 \\ \hat{\beta}_{1} & 0.658 & 0.020 & 0.658 & 0.034 & 0.640 & 0.049 \\ \hat{f}_{0} & 0.278 & 0.020 & 0.271 & 0.036 & 0.302 & 0.068 \\ \hat{\gamma}_{1} & 0.520 & 0.029 & 0.531 & 0.048 & 0.550 & 0.079\end{array}$

Regression Estimates

\begin{tabular}{lcccccc} 
& Mean & SD & Mean & SD & Mean & SD \\
$\hat{\lambda}_{1}$ & 0.845 & 0.015 & 0.721 & 0.040 & 0.545 & 0.066 \\
$\hat{\beta}_{1}$ & 0.631 & 0.018 & 0.552 & 0.042 & 0.467 & 0.056 \\
$\hat{\gamma}_{0}$ & 0.278 & 0.019 & 0.274 & 0.040 & 0.237 & 0.061 \\
$\hat{\gamma}_{1}$ & 0.570 & 0.025 & 0.681 & 0.059 & 0.935 & 0.099 \\
\hline
\end{tabular}

Theoretical values: $\lambda_{1}=.88, \beta_{1}=.65, \gamma_{0}=.27, \gamma_{1}=.52$.

$N=100$

These parameters remained the same for each of the three scenarios. The measurement error variances, $\sigma_{v 1}^{2}$ and $\sigma_{v 2}^{2}$, were manipulated in the three scenarios: 1.0 for Case 1; 5.0 for Case 2; and 15.0 for Case 3 . We would expect the differences between OLS estimates and the Kalman filter estimates to increase as more measurement error is introduced into the observed variables.

The results from the three simulations are in Table 1. For Case 1 , the differences between the two estimation methods show that even when random error is quite small, OLS estimates are biased, while the maximum likelihood estimates closely match the true parameters. In particular, the $\hat{\gamma}_{1}$ estimate (.57) deviates from the true parameter in the regression model (.52). The direction of the bias in the independent variable measured without error depends on the correlation between it and the variable measured with error. Once a moderate amount of error is introduced into the observed variables (Case 2), the advantages of estimating, rather than ignoring, the error become even clearer. The bias in $\hat{\gamma}_{1}$ increases even further and the estimates for $\hat{\beta}_{1}$ and $\hat{\lambda}_{1}$ move further away from the true values. The Kalman filter estimates are very close to the true parameter estimates for all the variables.

Case 3 most dramatically demonstrates the virtues of the Kalman filter. All the estimated coefficients for OLS begin to deviate considerably 
from the true parameter estimates, while the Kalman filter estimates remain close to the true value. The ability to estimate and control for the noise in a dynamic model allows the Kalman filter to produce unbiased estimates of the true parameters.

Application 1: The Impact of Opinion about Nuclear Power on NRC Enforcement

Within American politics, there is growing interest in the relationship between public opinion and public policy (see Jacobs and Shapiro 1994 and Page 1994 for extended reviews). Often, in looking at the relationship between policy and opinion, researchers rely on single indicators of these two concepts, but for many issue areas, policy and opinion are not likely to be perfectly measured. For example, survey marginals of public opinion regarding policy issues are likely to contain measurement error due to sampling procedures. If these survey marginals are used as independent variables to explain policy, coefficient estimates are likely to be biased. Because the dynamic linear errors-in-variables models include measurement equations, sampling error can be incorporated into the analysis as measurement error. In order to show how the dynamic linear errors-in-variables method can address these measurement problems, I will examine the link between citizens' attitudes towards nuclear power during the 1980's (OpposeNP) and the impact of these attitudes on regulatory enforcement by the Nuclear Regulatory Commission (Enforce).

The model for this analysis is

$$
\begin{aligned}
\text { OpposeNP }_{t} & =\lambda_{1} \text { OpposeNP }_{t-1}+e_{t} \\
\text { Enforce }_{t} & =\beta_{1} \text { Enforce }_{t-1}+\gamma_{0} \text { OpposeNP }_{t}+\epsilon_{t}
\end{aligned}
$$

where time $t$ indexes quarters. The measurement equations are

$$
\begin{aligned}
\text { OpposeNP }_{t}^{*} & =\text { OpposeNP }_{t}+v_{1 t} \\
\text { Enforce }_{t}^{*} & =\text { Enforce }_{t}+v_{2 t} .
\end{aligned}
$$

The specification of the first transition equation for opinion about nuclear power is consistent with the general view that public opinion follows an autoregressive process (Page and Shapiro 1992, 61-62). The second transition equation is specified so that the level of enforcement by the NRC is influenced by public opinion.

The enforcement budget variable is the quarterly expenditures by the NRC on inspections of nuclear power plants and enforcement of regulations through hearings and fines. Although budgeting for the Enforcement Division of the NRC is not done quarterly, we can still expect covariation between spending and opinion since the NRC can increase 
its visibility at nuclear power plants and in the press by more vigilantly enforcing regulations when it senses growing public concern about nuclear safety. In other words, the NRC has some discretion over how and when it spends its budget. The enforcement budget data were collected from the Monthly Treasury Bulletin.

Data on attitudes towards nuclear power were collected quarterly by Cambridge Reports Inc. for most of the 1980s. The question used as an indicator of attitudes towards nuclear power was: 'I'm going to read you a list of proposals for dealing with the energy crisis, and I'd like you to tell me whether you generally favor or oppose each one. ... building more nuclear power plants." The analysis was run using quarterly data from the first quarter of 1981 to the third quarter of 1988, when CRI stopped collecting these data. ${ }^{5}$ In this analysis, the percent opposing nuclear power was used to measure quarterly attitudes. Therefore, more opposition to nuclear power should result in stricter enforcement by the NRC as it tries to prevent accidents that might further erode public confidence in the safety of nuclear power. ${ }^{6}$ The data used in the analysis are mean centered. ${ }^{7}$

The OLS analysis of the two transition equations is shown in Table 2. Opinion regarding nuclear power is influenced by past attitudes, as indicated by the .701 coefficient on the lagged value of opposition to nuclear power. In the second transition equation in which the relationship between policy and opinion is modeled, opinion has a positive, significant impact of .286 on the enforcement budget. However, this coefficient is likely to be biased downward because of sampling error in the quarterly surveys.

With the Kalman filter the size of this random error due to sampling could be estimated. Since the proportions opposing expansion of nuclear

\footnotetext{
${ }^{5}$ The data collected by Cambridge Reports Inc. were reprinted in Rosa and Dunlap 1994.

'With survey marginals, it is possible that missing data create a bias problem in estimation. In this case, the missing data (or don't knows) could be negatively correlated with dramatic events (e.g., Chemobyl) since people are probably less likely to choose these options when dramatic events occur. This could create an omitted variables problem and biased coefficient estimates. Since the missing data are not reported by Cambridge Reports, we do not know if missing data pose a problem in this case.

${ }^{7}$ The two series used in this analysis-enforcement and opposition to nuclear power-were tested for unit roots. For each series, the null of a unit root could not be rejected. However, the enforcement variable had a unit root with no drift, while the opposition to nuclear power variable had a unit root with drift. Therefore, it is unlikely that these series could be co-integrated. This was confirmed by conducting a Engle-Granger test for co-integration which indicated that the series were not cointegrated. It is therefore not necessary to specify an error correction model.
} 
TABLE 2. Ordinary Least Squares Extimates for Opinion about Nuclear Power and the NRC's Enforcement Budget

Dependent Variable: Oppose Nuclear Power

$\begin{array}{lcc}\text { Variable } & \text { Coeff } & \text { SE } \\ \text { OpposeNP }_{t-1} & 0.701 * & 0.104\end{array}$

Adj. $R^{2}=0.62$

$\mathrm{SE}$ of Estimate $=3.367$

Dependent Variable: NRC's Enforcement Budget

$\begin{array}{lcc}\text { Variable } & \text { Coeff } & \text { SE } \\ \text { Enforce }_{t-1} & 0.385 * & 0.159 \\ \text { OpposeNP }_{t} & 0.286 * & 0.142\end{array}$

Adj. $R^{2}=0.69$

$\mathrm{SE}$ of Estimate $=4.52$

Quarterly data from 1981:01 to 1988:03

* $p<.05$

power and the sample size of the surveys are known, however, sampling error can be calculated and simply added to the model as error variance in the indicators and need not be estimated. Beck (1990) uses the same technique in an analysis of presidential approval survey marginals. The formula for estimating the sampling error is $X_{t}\left(100-X_{t}\right) / N$, where $X_{t}$ is the percentage of respondents holding a particular point of view and $N$ is the sample size. ${ }^{8}$

The results of the maximum likelihood estimates of the dynamic linear errors-in-variables model are reported in Table 3. The estimate of the effect of the lagged indicator for opposition to nuclear power expansion is virtually the same as the regression estimate. The differences between the maximum likelihood and regression point estimates for the second transition equation are larger. The coefficient for the lagged enforcement latent variable is .476 as compared to .385 in the regression

\footnotetext{
This is the correct formula for calculating the sampling error if the survey is based on a simple random sample. Cambridge Reports, like most polling organizations, did not rely on a simple random sample. The formula for the simple random sample is likely to underestimate the true sampling error. The correct sampling error could be calculated, albeit with great difficulty. Such a calculation would require precise knowledge of the degree of stratification and clustering in each sample.
} 
TABLE 3. Maximum Likefihood Estimates for Opinion about Nudear Power and the NRC's Enforcement Budget

Transition Equation 1: Oppose Nuclear Power

$\begin{array}{lcc}\text { Variable } & \text { Coeff } & \text { SE } \\ \text { OpposeNP } & 0.718 * & 0.127\end{array}$

Transition Equation 2: NRC's Enforcement Budget

$\begin{array}{llc}\text { Variable } & \text { Coeff } & \text { SE } \\ \text { Enforce }_{t-1} & 0.476 * & 0.237 \\ \text { OpposeNP }_{t} & 0.342 * * & 0.196\end{array}$

Error Variance for Transition Equations

\begin{tabular}{lll}
$\sigma_{e}^{2}$ & $4.589 *$ & 0.404 \\
$\sigma_{e}^{2}$ & $3.467 *$ & 1.721 \\
\hline
\end{tabular}

Quarterly date from 1981:01 to 1988:03

$* p<.05, * * p<.1$

analysis. ${ }^{9}$ The regression estimate for the coefficient for the opposition to expansion of nuclear power is .286 while the estimate from the maximum likelihood model is .342 . Despite the fact that the regression estimates consistently deviate from the maximum likelihood point estimates, the confidence intervals around the maximum likelihood estimates contain the regression estimates. The observed differences in the parameter point estimates are not statistically significant in this case.

\section{Multiple Indicators}

The dynamic errors-in-variables model described above demonstrates how single indicators are used to measure latent concepts. The analysis can be extended to include multiple indicators in the measurement model for the state vectors. The advantages of the multiple indicator approach are more efficient parameter estimates and the means to examine more systematically our measurement of theoretical concepts.

The extension from the single indicator model described above to

9I checked the residuals for signs of heteroskedasticity and did not detect 2 problem. Besed on some simulations in which I introduced heteroskedastic errors, the effect of heteroskedasticity in the dynamic linear errorb-in-variables setup is to inflate the standard errors, but not to introduced bias. 
a multiple indicator measurement model is fairly simple. If we return to the general model described in matrix form in equations (4) and (5), the transition model remains the same. The measurement model must be extended to include additional measures of the latent state variable. In the case of a three-indicator model for each of the state vectors, the new measurement model is

$$
\left[\begin{array}{l}
u_{i t}^{*} \\
u_{2 t}^{*} \\
u_{3 t}^{*} \\
x_{1 t}^{*} \\
x_{2 t}^{*} \\
x_{3 t}^{*}
\end{array}\right]=\left[\begin{array}{r}
u_{t} \\
\alpha_{2} u_{t} \\
\alpha_{3} u_{t} \\
x_{t} \\
\beta_{2} x_{t} \\
\beta_{3} x_{t}
\end{array}\right]+\left[\begin{array}{l}
v_{1 u t} \\
v_{2 u t} \\
v_{3 u t} \\
v_{1 x t} \\
v_{2 x t} \\
v_{3 x t}
\end{array}\right]
$$

The coefficient of one of the indicators must be set equal to one in order to provide a metric for the state variable. In equation (6) I have set $\alpha_{1}=\beta_{1}=1$. The addition of these indicators requires additional parameters to estimate, but estimation of the parameters via the Kalman filter proceeds exactly as described above. The dimensions of the innovations matrix change, but the log likelihood is identical to the one described in the appendix.

\section{Comparing Multiple Indicator and Single Indicator Models}

Using simulated data, it is possible to see the advantages that a multiple indicator model can have over one with single indicators of the latent variables. Since both models can estimate the random error in the independent variables, both methods should produce unbiased estimates of the true parameters. However, because a model with multiple indicators contains more information, it should provide more efficient estimates of the model parameters.

For this simulation, I estimate a model with two latent variables, each of which is measured with three indicators. The true parameters for the transition equations are the same as in the preceding simulation for the single indicator model: $\lambda_{1}=.88, \beta_{1}=.65, \gamma_{0}=.27, \gamma_{1}=.52$. In this simulation, there are 100 cases and the model was estimated 100 times. The results of this simulation are then compared to a model in which each latent variable is measured with a single indicator, in Table 4.

As expected, both models provide unbiased estimates. The errors around these estimates increase as the amount of measurement error increases. For example, the standard deviation for $\gamma_{1}$ rises from .009 to .025 as the measurement error increases from 1.0 to 15.0. For each of the parameters, the standard deviation for the estimate is smaller for 
TABLE 4. Summary Estimates for Simulations of Latent Variables with Multiple Indicators and Single Indicators

\begin{tabular}{|c|c|c|c|c|c|c|}
\hline Error: & \multicolumn{2}{|c|}{ Case 1} & \multicolumn{2}{|c|}{ Case 2} & \multicolumn{2}{|c|}{ Case 3} \\
\hline$\sigma_{v 1}=\sigma_{v 2}$ & \multicolumn{2}{|c|}{1.0} & \multicolumn{2}{|c|}{5.0} & \multicolumn{2}{|c|}{15.00} \\
\hline \multicolumn{7}{|c|}{ Multiple Indicator Dynamic Linear Errors-in-Variables } \\
\hline - & Mean & SD & Mean & SD & Mean & SD \\
\hline $\bar{\lambda}_{1}$ & 0.883 & 0.009 & 0.881 & 0.018 & 0.876 & 0.025 \\
\hline $\bar{\beta}_{1}$ & 0.651 & 0.012 & 0.655 & 0.024 & 0.651 & 0.032 \\
\hline ro & 0.272 & 0.014 & 0.275 & 0.029 & 0.277 & 0.041 \\
\hline$\dot{\boldsymbol{\gamma}}_{1}$ & 0.524 & 0.018 & 0.524 & 0.040 & 0.533 & 0.053 \\
\hline
\end{tabular}

Single Indicator Dynamic Linear Errorg-in-Variables

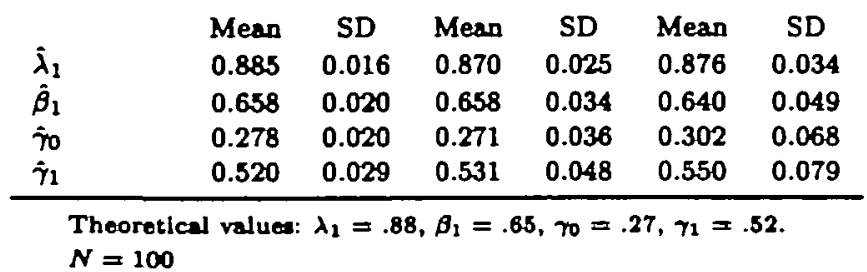

the multiple indicator model, demonstrating the efficiency gains it can provide.

The advantages of the multiple indicator model are not simply technical. The ability to estimate latent factors allows researchers to better link important concepts to their indicators when multiple indicators are available. The applications below illustrate how concepts like economic expectations and approval of government can be measured and analyzed by the dynamic linear errors-in-variables model.

Application 2: Presidential Approval and a Multiple-Indicator Model of Economic Expectations

Political scientists have spent a good deal of time trying to explain variation in presidential approval by using aggregate measures of economic conditions (for a thorough summary of the presidential approval literature, see Ostrom and Smith 1994) and have had limited success (Norpoth and Yantek 1983; Yantek 1988). The micro-level assumption behind the use of measures of aggregate economic conditions is that individuals are retrospective voters and evaluate presidents based on changes in their personal economic circumstances. According to this argument, individuals respond to changes in unemployment and inflation, and the effects of personal economic circumstances can be picked up by measures of aggregate economic conditions. Recently, however, this retrospective model 
has been challenged by both cross-sectional and time series analyses which show that prospective evaluations matter more than retrospective ones. MacKuen, Erikson and Stimson (1992) argue that, rather than responding to economic conditions, individuals evaluate the president's performance through their perceptions about the economy, and it is their expectations about the future state of the economy that matter most of all. MacKuen, Erikson and Stimson (1992) use the Index of Consumer Sentiment (ICS) and the components of the index to demonstrate that these aggregate measures of perceptions outperform direct measures of economic conditions in explaining presidential popularity.

This prospective model of presidential approval, which includes both measures of consumer sentiment and survey marginals for presidential approval, can easily and usefully be incorporated into the dynamic linear errors-in-variables setup. Estimation of a measurement model is a distinct advantage in analyzing a concept like economic expectations. Without a measurement model for the concept, one is forced to choose (using uncertain criteria) the "best" of several indicators of economic expectations.

MacKuen, Erikson and Stimson show that sociotropic rather than personal expectations about the economy have the greatest impact on evaluations of the president. Following their approach, I use the indexed response to two questions as indicators of people's expectations about future economic performance: 1) "Now turning to business conditions in the country as a whole- do you think that during the next 12 months we'll have good times financially, or bad times or what?" and 2) "Looking ahead, which would you say is more likely - that in the country as a whole we'll have continuous good times during the next 5 years or so, or that we will have periods of widespread unemployment or depression, or what?" MacKuen, Erikson and Stimson refer to these two questions as short-term and long-term business expectations. Responses to this question are compiled by the University of Michigan's Survey Research Center in its Survey of Consumers. ${ }^{10}$

The transition equations for the dynamic linear errors-in-variables model of presidential approval are

$$
\begin{aligned}
\text { BusExp } & =\gamma_{1} B u s E x p_{t-1}+e_{t} \\
\operatorname{PresApp}_{t} & =\beta_{1} \operatorname{PresApp}_{t-1}+\gamma_{0} B u s \operatorname{spp}_{t}+\gamma_{1} \text { Unem }_{t}+\gamma_{2} \operatorname{Inf}_{t}+\epsilon_{t}
\end{aligned}
$$

\footnotetext{
${ }^{10}$ Respondents are also asked about their personal financial expectations in the Survey of Consumers. I have excluded this measure from the analysis of economic expectations in order to specify a model that is consistent with MacKuen, Erikson and Stimson (1992), who argue that sociotropic evaluations matter more than personal. One could specify a measurement model for the more general concept "economic expectations" using all three indicators.
} 
The measurement equations are:

$$
\left[\begin{array}{c}
\text { Short-termBusExp } \\
\text { Long-termBusExpt } \\
\text { PresAppt }
\end{array}\right]=\left[\begin{array}{c}
B u s E x p_{t}^{*} \\
\alpha_{2} B u s E x p_{t} \\
{\operatorname{Pres} A p p_{t}}_{t}^{*}
\end{array}\right]+\left[\begin{array}{c}
v_{1_{t}} \\
v_{2_{t}} \\
v_{3_{t}}
\end{array}\right]
$$

The specification of the second transition equation closely follows that of MacKuen, Erikson and Stimson (1992). Unem $m_{t}$ is the change in the unemployment rate; and $I n f_{t}$ is the inflation rate measured by the Consumer Price Index. The specification of the transition equation for $B u s \operatorname{xp}_{t}$ helps insure that the model is identified and makes sense theoretically. Individuals' expectations are likely to be a function of past evaluations since their sources of information on the state of the economy and their interpretation of that information are likely to be fairly similar from one time period to the next.

Short-termBusExp * and Long-termBusExpt are the responses to the items asked in the Survey of Consumers. PresApp* is the percent approving of the president for the last survey taken in each quarter from 1960 to 1993 . The model is estimated quarterly, from the second quarter of 1960 to the fourth quarter of $1993 .{ }^{11}$

By using the results from the last survey in each quarter for presidential approval, the sampling error for each survey can be approximated and incorporated into the measurement equations of the dynamic linear errors-in-variables model. This is the same as the approach used in the analysis of opposition to nuclear power. One could tackle this problem differently through the use of quarterly averages. Quarterly averages should reduce the sampling error to a very small number. This was confirmed in some analyses that I conducted using the average for each of the quarters. Quarterly averages of survey marginals are not a luxury that many researchers have. To illustrate the more general approach and the techniques for handling sampling error, I present only the results from an analysis of the presidential approval measured by the last survey of the quarter.

I first estimate the two transition equations using OLS. For the OLS analysis, I used the short-term business expectations as an indicator of economic expectations and can therefore compare the results from OLS to the multiple indicator analysis. Table 5 shows the results of this OLS analysis. ${ }^{12}$ The significance of the lagged effects for the expectation

\footnotetext{
${ }^{11}$ Tests for unit roots were conducted on this data set. The presidential approval series did not have a unit root over this time period, therefore, the variables cannot be co-integrated.

${ }^{12}$ The regression model and Kalman filter model for presidential approval included dummy variables for the transition quarter between administrations.
} 
TABLE 5. Ordinary Least Squares Estimates for the Presidential Approval Model

Dependent Variable: Short-term Business Expectations

$\begin{array}{llc}\text { Variable } & \text { Coeff } & \text { SE } \\ \text { Short-termBusBIpt-1 } & 0.898 * & 0.104\end{array}$

Adj. $R^{2}=0.82$

$\mathrm{SE}$ of Eotimate $=13.95$

Dependent Variable: Presidential Approval*

$\begin{array}{lcc}\text { Variable } & \text { Coeff } & \text { SE } \\ \text { PresApp }_{t-1} & 0.660 * & 0.061 \\ \text { Short-termBusEsp } t & 0.082 * & 0.026 \\ \text { Change in Unemployment } & 0.139 & 0.246 \\ \text { Inflation } & -1.010 & 0.266\end{array}$

Adj. $R^{2}=0.62$

$\mathrm{SE}$ of Estimate $=7.59$

Quarterly data from 1960:2 to 1893:4

-The model was estimated with a dummy variable for each transition quarter between administrations. The coefficient estimate for the dummy varlable is not shown.

$\bullet p<.05$

equation is not surprising. The approval equation mirrors the results of Mackuen, Erikson and Stimson and shows that people's perceptions of the economy have a more reliable effect on presidential approval than do measures of real economic conditions. With short-term business expectations in the model, the coefficient for the change in unemployment has the wrong sign and is not significant.

Estimation of the dynamic linear errors-in-variables model for presidential popularity via the Kalman filter further confirms that expectations about the economy have a more reliable effect on presidential approval than current economic conditions do (Table 6). The estimates for the dynamic linear errors-in-variables model do suggest that the regression estimate for the lagged effect of short-term business expectations is attenuated. The regression estimate $(.90)$ is lower than that of the dynamic linear errors-in-variables model $(.936) .^{13}$ In the second

\footnotetext{
${ }^{13}$ Because the metric for the latent variable, $B u E E_{x p_{t}}$, is set equal to short-term business expectations and this variable is used as the indicator in the regression analysis, it is meaningful to compare the relative size of the coefficients from the two models.
} 
TABLE 6. Maximum Likefihood Extimates for the Presidential Approval Model

Transition Equation 1: Business Expectations

\begin{tabular}{|c|c|}
\hline Variable & Coeff \\
\hline Business Expectations $t-1$ & $0.936^{*}$ \\
\hline
\end{tabular}

Transition Equation 2: Presidential Approval

$\begin{array}{lrc}\text { Variable } & \text { Coeff } & \text { SE } \\ \text { Presidential Approval }_{t-1} & 0.682^{*} & 0.061 \\ \text { Business Expectations } & 0.105^{*} & 0.029 \\ \text { Change in Unemployment } & -1.098 & 1.016 \\ \text { Inflation } & -0.294 & 0.272\end{array}$

Error Variance for Transition Equations

$\begin{array}{lrr}\sigma_{9}^{2} & 131.240^{*} & 20.308 \\ \sigma_{a}^{2} & 51.977^{*} & 6.961\end{array}$

Measurement Equations

\begin{tabular}{lll} 
Short-term BusExp & $1.000^{*}$ & - \\
Long-termBusExp & 0.628 & 0.018 \\
PresApp & $1.000^{*}$ & - \\
\hline
\end{tabular}

Quarterly date from 1960:2 to 1993:4

-Indicatea a fixed parameter.

$-p<.05$

transition equation, the $B_{u s} x_{x p}$ variable is positively related to presidential approval, but a comparison of the regression estimate (.08) to the Kalman filter estimates (.105) shows that the regression estimate is somewhat lower. None of these differences are statistically significant, however. The confidence interval around the maximum likelihood estimate contains the regression estimate, and vice versa. In this analysis, the coefficients for inflation and the change in unemployment both have the correct sign although neither is significant.

The measurement equations also provide some information to help in the development and testing of theories. In this case, the coefficient between the indicator and the latent factor is significant, confirming that people's expectations about the economy form a single dimension and do not readily divide into a short and long-term component. 
Application 3: A Multiple-Indicator Moded of Approval and Economic Expectations

The analysis above can be extended to include a multiple indicator model for approval as well as business expectations. To do this, I specify a model to explain the effects of the economy on people's general approval of government, not just on their evaluation of the president. This can be accomplished by adding an indicator of citizen's approval of Congress to the measurement model so that both congressional approval and presidential approval are thought to be caused by a single latent approval variable. Thus, the dynamic linear errors-in-variables allows us to study concepts that are not readily measured by a single indicator.

The congressional approval series is constructed from a series of polls described in Magleby and Patterson (1994). The results from these polls were combined into a single series using the techniques developed by Stimson (1991) to estimate policy mood. The data are quarterly estimates of congressional approval from 1977 to $1993 .{ }^{14}$

The effects of the economy on this more general approval latent variable should be similar to those estimated for the presidential approval series. I use the same measures of business expectations, unemployment, and inflation to explain the variation in the general approval variable.

The transition equations are the same as in the presidential approval example reported above. The measurement models include congressional approval as an indicator of approval and are

$$
\left[\begin{array}{c}
\text { Short-termBusExpt } \\
\text { Long-termBusExpt } \\
\text { PresApp } p_{t}^{*} \\
\text { CongApp }
\end{array}\right]=\left[\begin{array}{r}
B u s E x p_{t}^{*} \\
\alpha_{2} B u s E x p_{t} \\
A p_{t} \\
\beta_{2} A p p_{t}
\end{array}\right]+\left[\begin{array}{l}
v_{1 t} \\
v_{2 t} \\
v_{3 t} \\
v_{4 t}
\end{array}\right]
$$

The results of the model are in Table 7 . In the measurement model, the loading of the congressional approval indicator on the approval factor is .971 and significant. This suggests that both indicators of approval are explained by an single underlying approval. The loading for the long-term business expectations on the underlying factor is .578 and also significant. As in the analysis of presidential approval, business expectations seem to form a single underlying expectations factor.

The transition equation for the approval model shows that the coefficient for lagged approval (.664) is significant and that the business expectations coefficient (.140) has a positive and significant impact on the general approval latent variable. The coefficients for the change in

\footnotetext{
${ }^{14}$ I am grateful to Jim Stimson and Mark Watts for supplying me with the congressional approval series.
} 
TABLE 7. Maximum Liketihood Extimates for the General Approval Moded

Transition Equation 1: Business Expectations

$\begin{array}{llc}\text { Variable } & \text { Coeff } & \text { SE } \\ \text { Business Expectations }-1 & 0.882^{*} & 0.057\end{array}$

Transition Equation 2: Approval

$\begin{array}{lcc}\text { Variable } & \text { Coeff } & \text { SE } \\ & & \\ \text { Approval }_{t-1} & 0.664^{*} & 0.080 \\ \text { Business Expectations } & 0.140^{*} & 0.038 \\ \text { Change in Unemployment } & -1.098 & 0.620 \\ \text { Inflation } & 0.509 & 0.292\end{array}$

Error Variance for Transition Equations

$\begin{array}{lrr}\sigma_{\varepsilon}^{2} & 154.005^{*} & 31.661 \\ \sigma_{\varepsilon}^{2} & 35.075^{*} & 7.671\end{array}$

Measurement Equations

\begin{tabular}{lll} 
Short-termBuEExpt & $1.000^{*}$ & - \\
Long-termBus $E x p_{t}$ & $0.578^{*}$ & 0.027 \\
PresApp $_{t}$ & $1.000^{*}$ & - \\
CongApp & $0.971^{*}$ & 0.068 \\
\hline
\end{tabular}

Querterly data from 1977:2 to 1993

-Indicates a fixed parameter.

$-p<.05$

unemployment and inflation are not significant at the .05 level. The unemployment coefficient has the correct sign but the inflation coefficient does not. Overall, the results for the general approval factor are similar to those from presidential approval-people's sociotropic and prospective evaluations of the economy have a significant impact on their evaluations of government, and these attitudes about the economy have more reliable effects than economic conditions do.

\section{Conclusion}

Despite the limited attention that time series analysts have devoted to measurement issues, the current simulations of the dynamic linear errorsin-variables model demonstrate that ignoring measurement error can be 
costly. Even with modest amounts of measurement error, parameter estimates are likely to be biased, and they get much worse as error increases.

Because the dynamic linear errors-in-variables model can incorporate multiple indicators of latent concepts, it can improve the efficiency of parameter estimates. This was evident in the simulation that compared a multiple indicator model to one using single indicators of latent variables. More importantly, the use of a multiple indicator model may free a researcher from having to choose only one of several potential indicators to represent a latent concept such as expectations about the economy or approval of government. With the dynamic linear errors-invariables model, it is possible to use all the available information from the indicators and specify a measurement model that links the indicators to the latent concepts.

In the substantive examples, a comparison of OLS to the maximum likelihood estimates did not show significant bias in the OLS estimates. Nonetheless, the analysis of the impact of opinion regarding nuclear power on regulatory enforcement and the consequences of economic perceptions on approval are important in demonstrating how measurement error can be addressed. The case of NRC enforcement and public opinion shows how knowledge about sampling error can be incorporated into the analysis using the measurement model in the dynamic linear errors-invariables model. The multiple indicator models demonstrate that more complex measurement of key concepts for both dependent and independent variables is possible.

Although the dynamic linear errors-in-variables model used here was fairly simple, the model is flexible enough to handle a variety of specifications. For example, the structure that was imposed on the independent variable through the additional transition equation is not required. The model can also be estimated with more complex lag structures, and laglength tests can be conducted by comparing log-likelihood ratios. Thus, attention to measurement error in time series analysis can assist political scientists in better estimating a variety of complex and simple models that implicitly rely on latent concepts, but are typically modeled using only noisy indicators of these concepts.

\section{APPENDIX: THE KALMAN FILTER}

In order to use the Kalman filter equations to estimate the unknown parameters for the dynamic linear errors-in-variables model, it is convenient to write equations (4) and (5) in a slightly more compact form. For times $t=1,2, \ldots$, the state vector is $\mathrm{X}_{t}=\left(u_{t}, x_{t}\right)^{\prime}$, with covariance matrix $P_{t}$, the measurement vector is $\mathbf{m}_{t}=\left(u_{t}^{*}, x_{t}^{*}\right)^{\prime}$, the vector of shocks 
is $w_{t}=\left(e_{t}, \epsilon_{t}\right)^{\prime}$, and the vector of measurement errors is $r_{t}=\left(v_{u t}, v_{x t}\right)^{\prime}$. The dynamic linear errors-in-variables model can be written as

$$
\begin{aligned}
& \mathbf{X}_{t}=\mathbf{B X} \mathbf{X}_{t-1}+\mathbf{G w}_{t} \\
& \mathbf{m}_{t}=\alpha \mathbf{X}_{t-1}+\mathbf{r}_{t}
\end{aligned}
$$

where

$$
\mathbf{B}=\left[\begin{array}{cc}
\lambda_{1} & 0 \\
\gamma_{0} \lambda_{1} & \beta_{1}
\end{array}\right] \quad \mathbf{G}=\left[\begin{array}{cc}
1 & 0 \\
\gamma_{0} & 1
\end{array}\right] \quad \alpha=\left[\begin{array}{ll}
1 & 0 \\
0 & 1
\end{array}\right] .
$$

The covariance matrices of the disturbances are

$$
\boldsymbol{\Sigma}_{w w}=\left[\begin{array}{cc}
\sigma_{e}^{2} & 0 \\
0 & \sigma_{\epsilon}^{2}
\end{array}\right] \quad \boldsymbol{\Sigma}_{r r}=\left[\begin{array}{cc}
\sigma_{v_{*}}^{2} & 0 \\
0 & \sigma_{v_{z}}^{2}
\end{array}\right] .
$$

To begin the filter, starting values $\mathbf{X}_{0}$ must be provided for the state vector and its covariance matrix $\mathbf{P}_{\mathbf{0}}$. There are different strategies for choosing starting values. For the state vector, previous values of the observed variables are often available and provide good starting values. Using the average of a stationary series is also an option. The covariance matrix can be set to relatively high values, indicating a high degree of uncertainty about prior information. Or the average values of $\mathbf{P}_{\boldsymbol{t}}$ from one pass through the Kalman filter can be used as the initial conditions.

Given $\mathbf{X}_{0}, \mathbf{P}_{0}$, do for $t=1, \ldots, T:$ (1) predict $\mathbf{X}_{t}$ from its known previous value,

$$
\mathbf{X}_{t \mid t-1}=\mathbf{B X}_{t-1 \mid t-1}
$$

(2) calculate the variance of $\mathbf{X}_{t}$ from updated previous variance and from prior information on $\boldsymbol{\Sigma}_{w w}$ and $\mathbf{G}$,

$$
\mathbf{P}_{t \mid t-1}=\mathbf{B} \mathbf{P}_{t-1 \mid t-1} \mathbf{B}^{\prime}+\mathbf{G} \boldsymbol{\Sigma}_{\boldsymbol{w} w} \mathbf{G}^{\prime} ;
$$

(3) calculate innovations by subtracting the values of the observed variables from the predicted values,

$$
\eta_{t \mid t-1}=m_{i}^{*}-\alpha \mathbf{X}_{t \mid t-1}
$$

(4) form the information matrix $H_{t}$ from the estimated variance, $P_{t}$,

$$
\mathbf{H}_{t}=\alpha \mathbf{P}_{t \mid t-1} \alpha^{\prime}+\Sigma_{r \boldsymbol{r}}
$$

(5) update $\mathbf{X}_{t}$, given current information about innorations, $\alpha^{\prime} \mathbf{H}_{t-1}^{-1} \eta_{t}$,

$$
\mathbf{X}_{t \mid t}=\mathbf{X}_{t \mid t-1}+\mathbf{P}_{t \mid t-1}\left(\alpha^{\prime} \mathbf{H}_{t-1}^{-1} \eta_{t}\right)
$$


(6) update $\mathbf{P}_{t}$, given $\alpha$ and $\mathbf{H}$,

$$
\mathbf{P}_{t \mid t}=\mathbf{P}_{t \mid t-1}-\mathbf{P}_{t \mid t-1} \alpha^{\prime} \mathbf{H}_{t-1}^{-1} \alpha \mathbf{P}_{t \mid t-1}
$$

\section{REFERENCES}

Aigner, Dennis, C. Hsiao, A. Kapteyn, and T. Wansbeek. 1984. "Latent Variables in Econometric Time-Series." In Handbook of Econometrics, Z. Griliches and M. Intriligator (eds.), Amsterdam: North-Holland.

Aoki, Masanao. 1990. State Space Madeling of Time Series. Berlin: SpringerVerlag.

Beck, Nathaniel. 1990. "Estimating Dynamic Models Using Kalman Filtering." Political Analysis 1:121-56.

Geweke, John. 1977. "The Dynamic Factor Analysis of Econometric TimeSeries." In Latent Variables in Socio-Economic Madelse, Dennis J. Aigner and Arthur S. Goldberger (eds.), Amsterdam: North-Holland.

Ghosh, Damanyanti. 1989. "Maximum Likelihood Estimation of the Dynamic Shock-Error Model." Joumal of Econometrics 41:121-43.

Harvey, Andrew C. 1989. Forecasting, Structural Time Series Madels, and the Kalman Filter. Cambridge: Cambridge University Press.

Kellstedt, Paul, Gregory E. McAvoy, James A. Stimson. 1996 "Dynamic Analysis with Latent Constructs." Political Analysis 5:113-50.

Jacobs, Lawrence R, and Robert Shapiro. 1994. "Studying Substantive Democracy." PS: Political Science and Politics 27.1:9-17.

Lomba, Jaime Terceiro. 1990. Estimation of Dynamic Econometric Models with Errors in Variables. Berlin: Springer-Verlag.

Maravall, Agustin. 1979. Identification in Dynamic Shock-Error Models. New York: Springer-Verlag.

Maravall, Agustin, and Dennis J. Aigner. 1977. "Identification of the Dynamic Shock-Error Model: The Case of Dynamic Regression." In Latent Variables in Socio-Economic Models, Dennis J. Aigner and Arthur S. Goldberger (eds.), Amsterdam: North-Holland.

MacKuen, Michael B., Robert S. Erickson, and James A. Stimson. 1992. "Peasants or Bankers? The American Electorate and the U.S. Economy." American Political Science Review 86:597-611.

Magleby, David B., and Kelly D. Patterson. 1994. "Congressional Reform (The Polls-Poll Trends)." Public Opinion Quarterly 58:419-28.

Norpoth, Helmut, and Thom Yantek. 1983. "Macroeconomic Conditions and Fluctuations of Presidential Popularity: The Question of Lagged Effects." American Journal of Political Science 27:785-807.

Nowak, Eugene. 1993. "The Identification of Multivariate Linear Dynamic Errors-in-Variables Models." Journal of Econometrics 59:213-27. 
Page, Benjamin. 1994. "Democratic Responsiveness: Untangling the Link between Public Opinion and Policy." PS: Political Science and Politics 27:25-29.

Page, Benjamin I., and Robert Y. Shapiro. 1992. The Rational Public: Fifty Years of Trends in Americans' Policy Preferences. Chicago: University of Chicago Press.

Ostrom, Charles W., Jr., and Renée M. Smith. 1994. "Error Correction, Attitude Persistence, and Executive Rewards and Punishments: A Behavioral Theory of Presidential Approval." Political Analysis 4:127-83.

Rosa, Eugene, and Riley E. Dunlap. 1994. "Three Decades of Public Opinion (The Polls-Poll Trends)." Public Opinion Quarterly 58:295-325.

Scherrer, W., M. Deistler, M. Kopel, and W. Reitgruber. 1991. "Solution Sets for Linear Dynamic Errors-in-Variables Models." Statistica Neerlandica 45:391-404.

Solo, V. 1986. "Identifiability of Time Series with Errors in Variables." In Essays in Time Series and Applied Processes, J. Gani and M. B. Preistley (eds.), Sheffield: Applied Probability Trust, Journal of Applied Probability, special volume $23 \mathrm{a}$.

Stimson, James A. 1991. Public Opinion in America: Moods, Cycles, and Suings. Boulder: Westview Press.

Watson, Mark W., and Robert F. Engle. 1983. "Alternative Algorithms for the Estimation of Dynamic Factor, MIMIC and Varying Coefficient Regression Models." Journal of Econometrics 23:385-400.

Williams, John T., and Michael D. McGinnis. 1992. "The Dimension of Superpower Rivalry: A Dynamic Factor Analysis." Journal of Conflict Resolution 36:68-118.

Yantek, Thom. 1988. "Polity and Economy under Extreme Conditions." American Journal of Political Science 32:196-216. 